\title{
Site Conditions for Astronomy at the South Pole
}

\author{
J.W.V. Storey ${ }^{a}$, M.C.B. Ashley ${ }^{a}$, M.G. Burton ${ }^{a}$ and M.A. Phillips ${ }^{a}$ \\ ${ }^{a}$ Joint Australian Centre for Astrophysical Research in Antarctica, \\ School of Physics, University of New South Wales, Sydney NSW 2052, Australia
}

\begin{abstract}
We discuss the site conditions for astronomy at the South Pole and over the Antarctic plateau. We find that these conditions are the most favorable on Earth for sensitive observations at thermal infrared and sub-millimeter wavelengths. We further discuss plans to develop infrared facilities to exploit this potential.
\end{abstract}

Keywords: Antarctica, Site Testing, Astronomy, Infrared, Seeing

\section{INTRODUCTION}

The Antarctic plateau provides unique conditions on the Earth for the conduct of observational astronomy. The air is thin, dry and cold, with stable weather; these attributes all offer gains to the observational astronomer. The conditions are quite different to those experienced at Antarctic coastal locations, which are frequently subject to violent storms.

The plateau is over $3,000 \mathrm{~m}$ in elevation, rising to more than $4,000 \mathrm{~m}$ at Dome A. An average year-round temperature of $-50^{\circ} \mathrm{C}$, falling to $-90^{\circ} \mathrm{C}$ at times, vastly reduces the thermal background in the near-IR. The precipitable water vapour content of the air is typically around $250 \mu \mathrm{m}$ and can fall below $100 \mu \mathrm{m}$, opening up new windows in the infrared and sub-millimeter regimes to ground-based observation. The lack of a diurnal temperature cycle and the low wind speeds on the highest parts of the Antarctic plateau provide conditions of extraordinarily stability, benefiting a wide range of observational programs.

Taken together these conditions provide for an unsurpassed observing environment for ground-based astronomy across wide ranges of the electromagnetic spectrum. The new science this can engender will be significant. ${ }^{1}$ In particular, it will allow us to pursue 'formation studies' through new observations in the infrared to millimeter spectral range. This includes the study of events such as the formation of galaxies, the birth of the first stars in them and their subsequent evolution, the life cycle of the interstellar medium and the formation of individual stars and planets in our Galaxy. There are three primary reasons why this is so: the continuum emission from these events peaks in the IR, their dominant cooling lines occur across this spectral range, and the cosmic microwave background peaks in the millimeter.

\section{NEAR-IR CONDITIONS}

The most sensitive observations that can be made will be in the $2.3-2.5 \mu \mathrm{m}$ ' $\mathrm{K}$-dark' window. Here airglow emission is virtually absent, and the thermal emission from sky and telescope is dramatically reduced in Antarctica. Measured background levels are only an order of magnitude higher than that of the zodiacal emission, the fundamental limit for all inner Solar System observatories. This itself is also at a minimum near these wavelengths.

Of particular scientific interest will be exploiting the $\mathrm{K}$-dark window for deep surveys, e.g., for faint stars, embedded star-forming clusters and for proto-galaxies. For instance, for redshifts in the range $z=3-10$, where galaxy formation occurs, the peak of the mean stellar spectrum is shifted into the near-IR. However, while the absolute

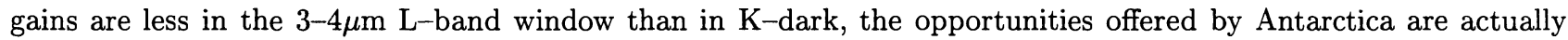
greater at the longer wavelength. This is a result of the combination of the reduced background, the wider bandpasses that can be employed than at temperate sites and the improved stability of the atmosphere. Only limited work has been done in L-band to date. Complete population censuses of star forming regions will be possible, the $3-4 \mu \mathrm{m}$ band making discrimination of embedded objects with disks from background reddened stars much easier than at $2 \mu \mathrm{m} .{ }^{3}$

Other author information: (Send correspondence to jwvs)

E-mails: J.Storey@unsw.edu.au, M.Ashley@unsw.edu.au, M.Burton@unsw.edu.au, A.Phillips@unsw.edu.au 

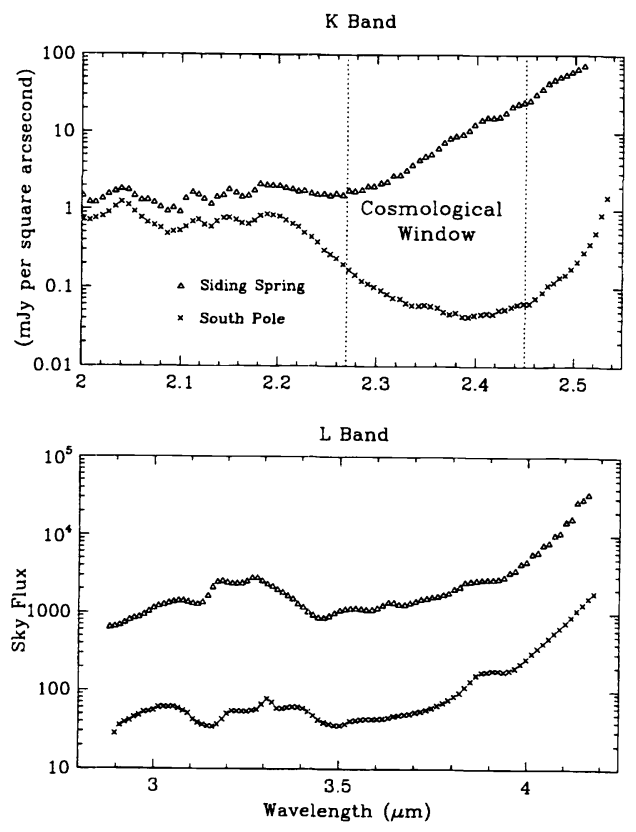

Figure 1. Sky fluxes in mJy per square arcsecond measured at the South Pole in winter in the K $(2-2.4 \mu \mathrm{m})$ and L $(3-4 \mu \mathrm{m})$ bands, ${ }^{2}$ compared with those at Siding Spring Observatory in Australia. Siding Spring can be considered typical of other temperate sites such as Kitt Peak. The ' $\mathrm{K}$-dark', or 'cosmological window', is indicated.

In 1994 the University of New South Wales and CARA, the Center for Astrophysical Research in Antarctica, began a collaboration to determine the site characteristics at the South Pole. Their first experiment was a nearinfrared sky monitor, the 'IRPS', measuring the brightness of the sky emission from 2 to $4 \mu \mathrm{m}$ over the winter. The IRPS was modified from the Anglo Australian Observatory's first infrared photometer of the early 80's. Working in parallel with CARA's SPIREX telescope, both the existence of the postulated 'cosmological window' at $2.4 \mu \mathrm{m}$ was demonstrated, and a deep window from $3-4 \mu \mathrm{m}$ discovered. These particular results can be quantified as follows (see Fig. 1):

- Sky background in K-dark window $(2.27-2.45 \mu \mathrm{m})$ as low as $\sim 100 \mu \mathrm{Jy} \operatorname{arcsec}^{-2}(20-100$ times less than at temperate sites). ${ }^{2,4}$

- Sky background in L-band $(3-3.8 \mu \mathrm{m}) \sim 100 \mathrm{mJy} \operatorname{arcsec}^{-2}(\sim 20$ times less than at temperate sites $){ }^{2}$

An interesting demonstration of the low background at $2.4 \mu \mathrm{m}$ over the plateau comes from an all-sky map produced by the IRPS, obtained as the DC sky signal in a $4^{\circ}$ beam through the effective $5 \mathrm{~mm}$ diameter aperture of its 'telescope' (Fig.2) while performing 'sky dips' from zenith to horizon. The Galactic Plane is clearly visible, and the map compares very favorably with a similar image produced by the DIRBE instrument of the COBE satellite. ${ }^{5}$ The IRPS is effectively seeing the sky at $2.4 \mu \mathrm{m}$ as our eyes see it in the optical, with the Milky Way clearly visible against a dark background. From no other site on Earth would the Milky Way stand out like this at infrared wavelengths.

\section{MID-IR CONDITIONS}

By virtue of its location, the South Pole spends six months of the year in continuous daylight. At 10 and $20 \mu \mathrm{m}$ this represents perfectly workable conditions. By the nature of the available logistical support, it is far easier to operate through the summer months than in the winter. Reductions in background sky emission of $\sim 2.5$ times were expected due to the lower temperature, with further reductions possible due to the lower water vapor, aerosols and 

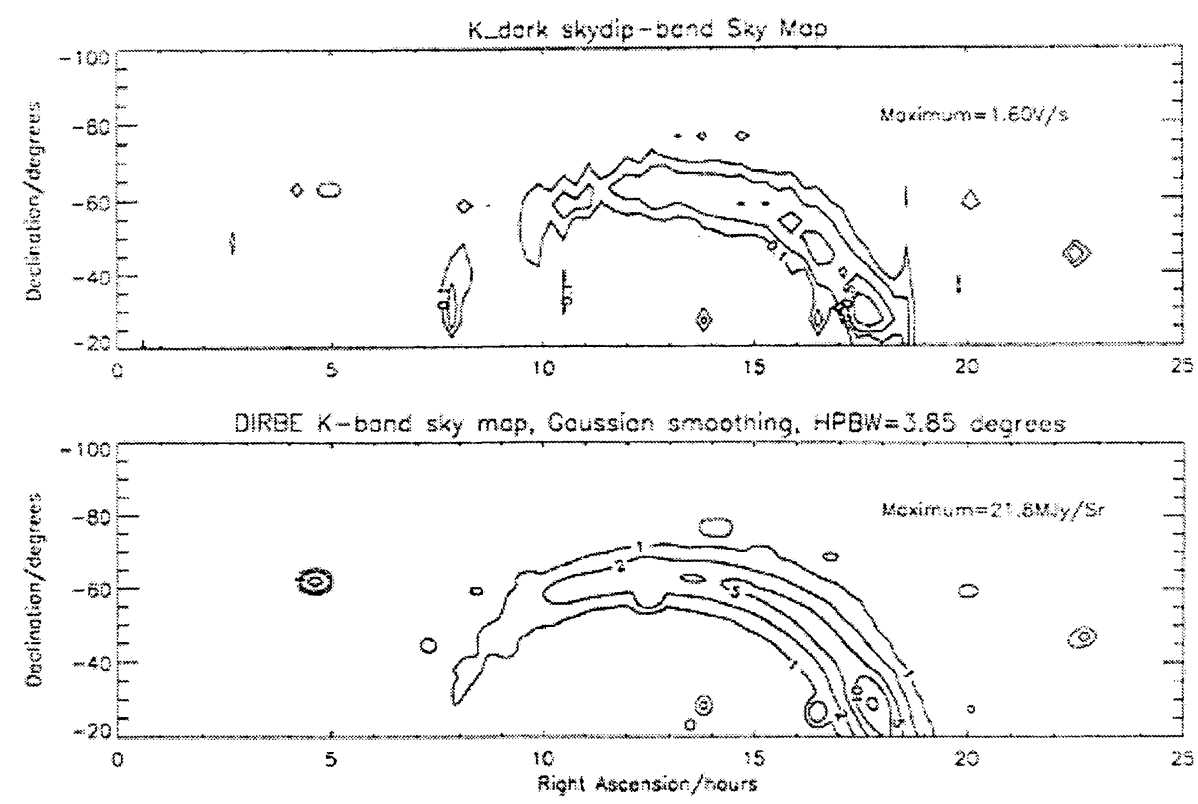

Figure 2. The Galactic Plane at $2.4 \mu \mathrm{m}$, as seen by the IRPS and COBE/DIRBE ${ }^{5}$ through a $4^{\circ}$ beam.

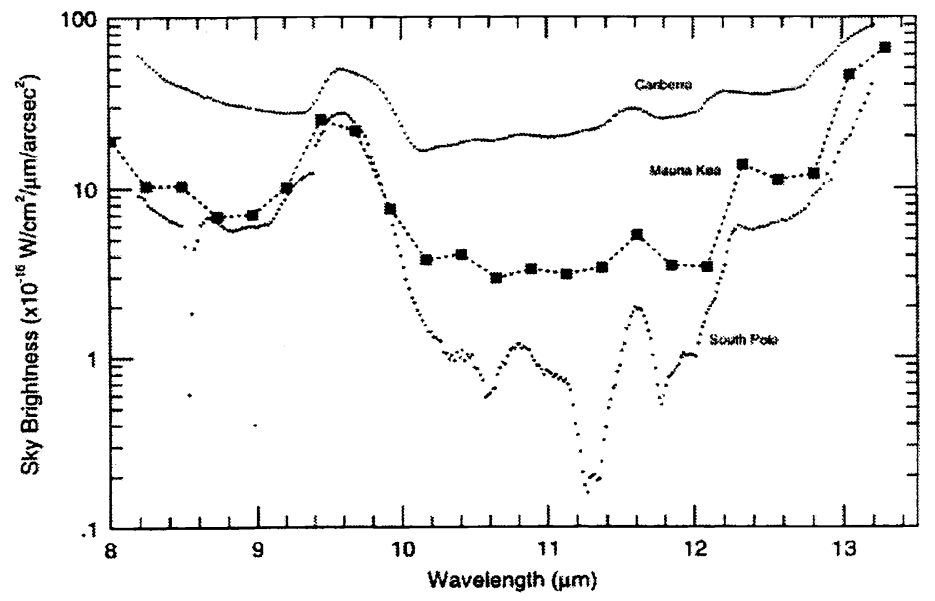

Figure 3. Sky fluxes measured at the South Pole in summer (day time) in N-band $(8-13 \mu \mathrm{m}){ }^{6}$ compared to Mauna Kea and Canberra in winter (night time). 
dust content of the atmosphere. On the other hand wind-blown ice crystals might act as both a source of increased background and of decreased stability.

To test these conjectures two sets of experiments have been undertaken. Firstly Dr. Craig Smith of the University College, UNSW, observed with his NIMPOL instrument at the South Pole during January 1996 daytime sky fluxes from $10-20 \mu \mathrm{m}$. Further data, extending out to $40 \mu \mathrm{m}$, were obtained during the 1997 winter by the SPIRAC camera (and telescope) run by CARA and NASA/GSFC. The specific results are as follows:

- Summer time sky background at $11.5 \mu \mathrm{m} \sim 35 \mathrm{Jy} \operatorname{arcsec}^{-2}$ ( $\sim 10$ times less than best values at temperate sites), with an anticipated minimum level of $\sim 1 \mathrm{Jy} \operatorname{arcsec}^{-2}$ in winter. ${ }^{6}$

- Sky backgrounds at $20 \mu \mathrm{m}$ reduced by a factor of $\sim 4$ times.

- Sky noise at $11.5 \mu \mathrm{m}$ restricted to frequencies below $0.2 \mathrm{~Hz}$ compared to $2 \mathrm{~Hz}$ at temperate sites. ${ }^{6}$

- Out to $40 \mu \mathrm{m}$ the transmission shows there is less than $250 \mu \mathrm{m}$ of precipitable $\mathrm{H}_{2} \mathrm{O}$ in the atmosphere, confirming previous determinations from the sub-millimeter. ${ }^{7}$

These results exceeded expectations. At $11.5 \mu \mathrm{m}$ the 'Craig window' is particularly clear, and much better than expected from simply the drop in temperature. Both the superb clarity of the atmosphere and its increased transmission contribute significantly to the gain.

\section{MILLIMETER AND SUB-MILLIMETER CONDITIONS}

The gains for millimeter and sub-millimeter astronomy stem from the improvement in the atmospheric transmission above the plateau. Many windows in the sub- $\mathrm{mm}$ band are opened up that have only been accessible before from airborne and spaced-based platforms. Equally significant is the stability of the atmosphere. The windows are open virtually continuously and the sky emission in them stable, allowing accurate background subtraction and flux calibration. Site testing results are as follows:

- Vastly improved atmospheric transmission in the sub-millimeter over temperate sites, with less than $250 \mu \mathrm{m}$ ppt $\mathrm{H}_{2} \mathrm{O}$ for half the time in winter, ${ }^{8}$ compared to best values of $\sim 1 \mathrm{~mm}$ elsewhere.

- Zenith opacity at $492 \mathrm{GHz}(610 \mu \mathrm{m})$ below 0.7 for half the time during winter and spring, and remaining below 1 for weeks at a time. ${ }^{8}$

- Zenith opacity in the millimeter, at $225 \mathrm{GHz}(1.3 \mathrm{~mm})$, less than 0.05 for over half the time, with sky noise more than 20 times smaller than good temperate sites in the winter. ${ }^{9}$

\section{SEEING}

The stable atmospheric conditions, tenuous air and absence of jet streams combine to produce conditions of superb clarity, or 'super-seeing'. Mitigating against these positive attributes, however, is the presence of a strong inversion layer which occurs on the most stable days of winter, when the air temperature can rise by $10^{\circ} \mathrm{C}$ in a few meters. This produces relatively poor ice-level seeing. However the degradation occurs almost entirely in the lowest $\sim 100 \mathrm{~m}$ of the atmosphere. Site testing results give:

- Mean winter temperature $-60^{\circ} \mathrm{C}$.

- Mean winter windspeed $8 \mathrm{~m} \mathrm{~s}^{-1}$ at bottom of boundary layer and $4 \mathrm{~m} \mathrm{~s}^{-1}$ at top of it.

- Median visual seeing on-ice $\sim 1.6^{\prime \prime}$.

- Median visual seeing at top of boundary layer $(\sim 190 \mathrm{~m}) 0.32^{\prime \prime}{ }^{10,11}$

- Median Fried parameter at ice level, $\mathrm{r}_{0}, \sim 6.4 \mathrm{~cm}$ at $5500 \AA$ and $\sim 37 \mathrm{~cm}$ at $2.4 \mu \mathrm{m} .{ }^{11}$ At the top of the boundary layer $\mathrm{r}_{0}$ is $\sim 28 \mathrm{~cm}$ and $\sim 1.6 \mathrm{~m}$, respectively. 
- The corresponding isoplanatic angle (after full adaptive optics correction of the boundary layer contribution) at $5500 \AA$ is $1-2^{\prime}$. This is much greater than at temperate sites, where it is typically only $10^{\prime \prime}$.

The seeing is thus of a quite different nature to that encountered at temperate latitudes, with a much larger isoplanatic angle and longer coherence times for the seeing cells. Low order adaptive optics systems will be able to recover most of the seeing within this isoplanatic angle.

The turbulence which causes seeing is closely correlated with the katabatic wind, and in particular the wind velocity gradient within the boundary layer. This suggests that the ice-level seeing at higher elevations on the plateau, especially on top of the ice Domes where the katabatic wind is absent, may be considerably smaller and approach that of the free atmosphere above the boundary level. Moreover the depth of the boundary layer decreases with elevation on the plateau. On top of the Domes a telescope on a raised platform may be above it entirely. This exciting prospect is the subject of an experiment with the AASTO (see §6.1).

\section{FUTURE WORK}

\subsection{The AASTO}

The South Pole is probably not the optimal site for astronomy on the plateau. It is directly under the auroral circle and also experiences a steady katabatic wind of $5-10 \mathrm{~m} / \mathrm{s}$. The best observing sites are expected to lie at the high points of the plateau, the $4,000 \mathrm{~m}$ Dome A and $3,200 \mathrm{~m}$ Dome C, where this wind is absent and the depth of the inversion layer less. How much better these sites are remains to be seen, but acquiring the necessary site-testing data from such uninhabited sites is difficult. Fortunately, the geophysical community have for many years been developing the 'Automated Geophysical Observatory', or AGO, ${ }^{12}$ which provides a suitable laboratory to perform the experiments. There are now six AGOs deployed across the plateau.

The AASTO, or 'Automated Astrophysical Site Testing Observatory' is, in effect, the seventh AGO and incorporates several small improvements over that design. It was built by Lockheed-Martin under contract to the University of New South Wales and the Australian National University. ${ }^{13,14}$ Like the AGOs, it is designed for field deployment from a ski-equipped LC-130 Hercules transport plane. It is a self-powered, self-heated autonomous laboratory. It is currently operational at South Pole station, and will later be deployed to remote, uninhabited sites on the high plateau. It is being fitted with a suite of astronomical site-testing instruments, so that potential observatory sites can be fully characterized over a wide range of wavelengths. The AASTO was formally opened by Senator Robert Hill, Australian Minister for the Environment and leader of the Senate, in January 1997.

After field testing the AASTO at the Pole in the 1998 and 1999 winters, it is planned to bring it first to Dome C, and then to Dome A. We aim to know how good the best observing site on the planet is by 2001.

\subsection{SPIREX/Abu}

SPIREX, the 'South Pole InfraRed EXplorer', is a 60-cm telescope built in 1994 by CARA in order to conduct observations in the $2.4 \mu \mathrm{m}$ 'cosmological window'. ${ }^{15,16}$ An agreement between the Universities of Chicago and Ohio State, NASA Goddard, the US National Optical Astronomy Observatory and the University of New South Wales has resulted in a project to upgrade SPIREX and install a state-of-the-art IR focal plane array (NOAO's 'Abu' camera, see paper by Fowler et al., these proceedings). UNSW's contribution includes the thermal control system, design and installation of new optics, and the addition of a CCD fast guider and tip-tilt secondary mirror to improve the tracking and pointing. This upgrade has taken place during the 1997/98 summer season at the Pole.

The system has been optimised for the thermal infrared, from $3-5 \mu \mathrm{m}$. For wide-field surveys at these wavelengths SPIREX/Abu is, despite its modest $60-\mathrm{cm}$ diameter mirror, the most powerful instrument on Earth. Of particular interest is the use of a tip-tilt secondary, allowing us to determine how much of the large isoplanatic patch above the plateau will be recoverable with the simplest form of wavefront correction. There are many projects that it is well suited to, including:

- Line imaging of embedded $\mathrm{HII}$ regions along the southern galactic plane in $\mathrm{Br} \alpha$, PAHs and $\mathrm{H}_{2}$ filters, to study the environment of the ionized, neutral and molecular mediums of massive star forming regions. 
- A survey of the Large Magallenic Cloud for massive star formation at L-band (3.5 $\mu \mathrm{m})$. Spending 1 hour per position, a $2^{\circ} \times 2^{\circ}$ region can be fully-sampled in a month of telescope time. Several thousand stars should be visible down to $\mathrm{L}=15.5$ magnitudes. While this only allows us to sample the top end of the mass distribution, it will now be possible to survey the LMC in a systematic manner for the regions where these stars form.

- The star formation history of the Universe is being probed through deep, pencil-beam surveys, of which the Hubble Deep Field (HDF) is the most notable example. A southern field (the HDF-S) is shortly to be obtained. SPIREX/Abu will be capable of complementary deep K- and L-band imaging to the optical bands of the HDFS. With 24 hours of on-source integration a detection threshold of 19.2 magnitudes through its $\mathrm{H}_{2} 2.4 \mu \mathrm{m}$ filter and 17.3 magnitudes through its $\mathrm{L}-$ band $3.5 \mu \mathrm{m}$ filter could be achieved. Based on the number counts in the $\mathrm{HDF}$ this would yield $\sim 40$ galaxies at $\mathrm{K}$ and $\sim 4$ at $\mathrm{L}$. These galaxies will typically have $\langle\mathrm{V}\rangle=22.5$, $\mathrm{V}-\mathrm{K}=3.5$ and a redshift $\mathrm{z}=0.6$. SPIREX/Abu would thus sample the upper end of the galaxy luminosity function, but with minimal uncertainties from extinction. However, it would also be sensitive to particularly red galaxies at high redshift; for instance an $\mathrm{E} / \mathrm{S} 0$ galaxy at $\mathrm{z}=1.4$ has an (unreddened) $\mathrm{V}-\mathrm{L} \sim 10$ and so would be detected by Abu if $\mathrm{V}<27$.

SPIREX/Abu also serves as a demonstrator of the scientific potential of the Antarctic plateau. While wide-field, deep, thermal infrared surveys are a particular niche this experiment can exploit, the work that a $60 \mathrm{~cm}$ telescope can do is limited. SPIREX/Abu is thus a forerunner for an intermediate size infrared telescope, a project we call 'SPIRIT'.

\subsection{SPIRIT}

The NSF is upgrading the Pole station so that it is capable of supporting some of the more ambitious scientific plans for it. One of these is for an intermediate-scale infrared facility. Australia is particularly interested in contributing to the development of such a facility, and has formulated plans for the 'South Pole InfraRed Imaging Telescope', or SPIRIT. ${ }^{17}$ This is envisaged as a 2.5 -m class infrared-optimised telescope capable of yielding near-diffraction limited images at $2.4 \mu \mathrm{m}$. It will be a wide-field telescope, instrumented with large format focal plane arrays, and operate primarily from $2-5 \mu \mathrm{m}$ and $8-30 \mu \mathrm{m}$. The first programs would involve surveys of star forming regions in these bands, to make a complete census of their embedded populations.

\section{SUMMARY}

The South Pole provides an excellent site for a wide range of astronomical observations across the infrared and millimeter wavebands. It is superior to any other ground based site currently in use for thermal infrared and sub-millimeter observations. Exploiting this advantage presents a number of interesting challenges, both logistical and technical. These include the use of low-order adaptive optics corrections schemes to remove the boundary layer contribution from the seeing. The Domes of the Antarctic plateau, where this boundary layer contribution is expected to be minimal, should provide the pre-eminent observing sites on the Earth.

\section{Acknowledgments}

We are grateful to numerous colleagues for their support and encouragement in our Antarctic endeavours, including especially those from CARA, the ANU and the UNSW. We particularly wish to thank Max Boccas, Rodney Marks and Ant Schinckel for their dedicated efforts in our research program, and Craig Smith, Al Harper and Sean Casey for allowing us to use unpublished data.

\section{References}

\section{REFERENCES}

1. M. Burton and twenty others Proc. Astron. Soc. Aust. 11, p. 127, 1994.

2. M. Ashley, M. Burton, J. Storey, J. Lloyd, J. Bally, J. Briggs, and D. Harper Pub. Ast. Soc. Pac. 108, p. 721, 1996.

3. K. Strom, S. Strom, S. Edwards, S. Cabrit, and M. Strutskie Ast. J. 97, p. 1451, 1989.

4. H. Nguyen, B. Rauscher, S. Severson, M. Hereld, D. Harper, R. Loewenstein, F. Mrozek, and R. Pernic Pub. Ast. Soc. Pac. 108, p. 718, 1996. 
5. COBE Diffuse Infrared Background Experiment Explanatory Supplement Version 2.0 COBE Ref. Pub. No. 95-A, NASA/GSFC, 1995.

6. C. Smith and D. Harper Pub. Ast. Soc. Pac., 1998.

7. S. Casey, "private communication," 1997.

8. R. Chamberlin, A. Lane, and A. Stark $A p J$ 476, p. 428, 1997.

9. R. Chamberlin and J. Bally Int. J. of IR \& MM Waves 16, p. 907, 1995.

10. R. Marks, J. Vernin, M. Azouit, J. Briggs, M. Burton, M. Ashley, and J. Manigault Ast. Ap. Supp. 118, p. 385, 1996.

11. R. Marks, J. Vernin, M. Azouit, J. Manigault, and C. Clevelin Ast. Ap. , 1998.

12. J. Doolittle in Lockheed Technical Report LMSC-F171145, Lockheed Missiles \& Space Co. Palo Alto, 1986.

13. J. Storey, M. Ashley, and M. Burton Pub. Ast. Soc. Aust. 13, p. 35, 1996.

14. J. Storey in Astrophysics from Antarctica, G. Novak and R. Landsberg, eds., ASP Conf. Series, 1998.

15. D. Harper in Astrophysics in Antarctica, D. Mullan, M. Pomerantz, and T. Stoner, eds., Am. Inst. Phys. Conf. ser. 198, p. 123, 1989.

16. M. Hereld in Infrared astronomy with arrays: the next generation, I. McLean, ed., Ast. Ap. Sci. Lib. 190, p. 248, 1994.

17. M. Burton, J. Storey, and M. Ashley, "Looking deep from the south pole: star formation in the thermal infrared," in Looking Deep in the Southern Sky, ESO workshop proceedings , 1998. 